\title{
Fundamental Relationships of Executive Functions and Physiological Abilities with Game Intelligence, Game Time and Injuries in Elite Soccer Players
}

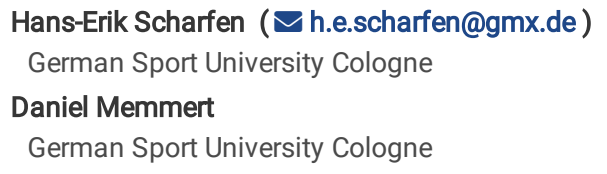

\section{Research Article}

Keywords: cognitive functions, high-performance athletes, physiological performance, talent prediction

Posted Date: March 31st, 2021

DOI: https://doi.org/10.21203/rs.3.rs-360885/v1

License: () (7) This work is licensed under a Creative Commons Attribution 4.0 International License. Read Full License

Version of Record: A version of this preprint was published at Applied Cognitive Psychology on October 25th, 2021. See the published version at https://doi.org/10.1002/acp.3886. 


\section{Abstract}

Objectives: The study's aim was threefold: examine 1) the relation between executive functions and coach-rated evaluation of game intelligence; 2) the predictive value of cognitive and athletic performance parameters concerning playing time, and 3) injuries to analyze fundamental associations of soccer expertise across all ages.

Design/Methods: A sample of 172 elite soccer players (12-34 years of age) performed tests assessing multiple-object tracking, working memory capacity, cognitive flexibility, and inhibition. Endurance-performance at the individual anaerobic threshold (IAT), ability to repeatedly perform intense exercises (RIEA) and maximal anaerobic performance parameters (squat jump, countermovement jump, drop jump, sprint) were also measured. Game intelligence game time and injury incidence were tracked.

Results: Working memory capacity, cognitive flexibility, and a total cognition score showed correlations with game intelligence, and the same parameter, along with selective attention and game intelligence, were also correlated with game time. Sprint and RIEA were connected with game time, whereas contact injuries only correlated with sprint, and noncontact injuries with sprint and performance-IAT.

Conclusion: Especially executive functions represent fundamental associations with game intelligence and game time across all age groups but not with injury incidences, whereas certain physiological abilities may contribute to more game time and less non-contact injuries depending on age.

\section{Introduction}

The prediction of talent across all ages is a crucial aspect of high-performance settings ${ }^{1}$. One of these settings is team and racket sports like elite soccer, where certain physiological and cognitive skills are required to operate on an extraordinary level. Identifying the principal mechanisms of high-performance of all age groups in this sport is of common interest as soccer is a largescale phenomenon with 265 million players around the world ${ }^{2}$. Hence, soccer associations and clubs need to find the players among the large amount with certain characteristics enabling them to succeed later or currently in their career on a professional level ${ }^{3}$. However, the combined predictive value of physiological and cognitive skills is still unclear ${ }^{4,5}$. Thus, the present study is unique as it aims to examine the relationship among both abilities and success of youth as well as adult elite soccer players.

Though, previous studies examined how certain objective performance parameters may have a prognostic value in isolation. The majority of these investigations had a monodisciplinary focus on physical (e.g. height and weight) or physiological (e.g. endurance and speed) determinants whereby the latter has been partially related to success in soccer (for reviews see ${ }^{4,5}$ ). However, it is well-known that success in a complex game like soccer depends on multidisciplinary, symbiotic aspects which needs to be investigated in longitudinal study designs according to best practice guidelines ${ }^{5,40-43}$. However, these guidelines have rarely been applied in previous studies ${ }^{5,40}$. Current models propose that the cognitive domain is an important part of this multidisciplinarity as well, besides physiological characteristics ${ }^{40-45}$. Thus recent studies established a promising association to elite performance ${ }^{10-13}$.

First studies represent encouraging value for talent identification but evidence on their predicational value, especially across several age groups is scarce as the majority of the investigations had a monophasic focus $5,7,14-16,28,40$. Specifically, a crucial subgroup of these cognitive skills are the executive functions defined as cognitive processes that regulate thought and action, especially in nonroutine situations $\mathbf{s}^{8,9}$. They include working memory, cognitive flexibility, and inhibitory control. Furthermore, best practice guidelines also propose the inclusion of coach-rated criteria which has been rarely conducted previously ${ }^{5}$.

A first attempt to analyze those coach-rated criteria for the cognitive domain showed that the coaches' assessment of game intelligence is moderately correlated with executive functions' objective parameters in adult elite soccer players ${ }^{16}$. Nevertheless, this interrelation has not been shown in adolescents yet, and the predictive value of a combination of these coach-rated- and objective cognitive parameters is still unknown.

Another line of research concerning performance data emphasizes that high injury-risks are associated with poor cognitive functioning ${ }^{17-19}$. Nevertheless, the predictive capabilities of behavioral data for real injuries and not only injury-risks need further evaluation ${ }^{20}$. More specifically, the differentiation between contact and noncontact injuries is relevant due to the contrasting underlying mechanisms and their distinct incidence numbers ${ }^{19}$.

However, by reviewing the literature on previous endeavors to predict talent and injuries, it becomes evident that the principal focus was 1) monodisciplinary by either predominantly examining the physiological (i.e. talent prediction) or cognitive domain (i.e. injury prediction) and 2) monophasic by either focusing or adults or adolescents. Although first investigations of the prognostic value of cognitive performance are promising this area is still in its infancy and also quite debatable ${ }^{33}$. Additionally, the predictive value of coach-rated assessments of this cognitive performance and its interrelation with objective cognitive parameters are mostly unknown and, previous examinations only included adults. Thus, using a multiphasic focus by integrating both age groups may also evaluate for the first time if certain relationships (e.g. executive functions and game time) are present across all tested age groups. Consequently, it could be analyzed whether fundamental associations exist for soccer expertise at every developmental phase. This would yield valuable insights in terms of a) a performance-needs analysis of current demands of high-performance in complex team sports like soccer and b) a more sophisticated understanding of the contribution of cognitive and physiological skills of expertise and talent to certain developmental stages $40,41,43,44$.

Based on the outlined literature gaps, the aim of the present study of adolescent and adult elite soccer players (i.e. 12-34 years of age) is threefold. Firstly, the interrelation of coach-rated game intelligence and objective assessments of executive functions is analyzed. Secondly, the prognostic capabilities of multidisciplinary and multiphasic performance data, including physiological and cognitive (objective and coach-rated) parameters, are examined in two regards: 1 ) in terms of future success defined as the playing time per player in games which is a commonly used performance indicator ${ }^{34}$ and 2 ) in terms of 
future injuries (i.e. contact and non-contact). Further, based on previous literature it is hypothesized that 1) game intelligence correlates with executive functions independently of age, 2) better physiological and cognitive skills correlate with more playing time and 3) fewer injuries independently of age.

More specifically, game intelligence, executive functions (i.e. working memory, cognitive flexibility, inhibition), and attention (multiple-object tracking) are analyzed based on their crucial role in elite soccer (game intelligence ${ }^{16}$; executive functions ${ }^{10-15}$; multiple-object tracking ${ }^{21,22}$ ). Physiological abilities are examined in terms of endurance performance with an incremental field test (i.e. performance at the individual anaerobic threshold (IAT) and a YoYo intermittent recovery test (i.e. maximal performance stage stating the ability to perform intense exercises repeatedly (RIEA)). As well as in terms of maximal anaerobic performance: with vertical jumps (i.e. squat jump (SJ), countermovement jump (CMJ), drop jump (DJ)), and with a 30-meter sprint. These measurements are included as they are important performance properties of high-level soccer players ${ }^{4,23-26}$ and are commonly examined in the talent prediction studies of the physiological domain ${ }^{4,5}$. Additionally, we are following several calls ${ }^{5,40-43}$ to conduct multidisciplinary, multiphasic and longitudinal research including coach-rated assessments and aim to expand previous findings ${ }^{16}$ in a similar study design with a larger population also including adolescents.

\section{Results}

Partial Correlations (Spearman's r) with their $95 \%$ confidence intervals and the sample sizes contributing to each correlation are stated in Tables 1,2 and 3. Bivariate correlations are presented in the appendix. A preliminary analysis showed that the slightly different performance level of the U23 compared to the other teams did not influence the results substantially.

\section{Game intelligence}

Working memory capacity, cognitive flexibility and the cognition score showed small to moderate correlations with game intelligence. In contrast, selective attention only showed a trend towards a meaningful correlation and inhibition showed no correlation at all. The partial correlation analysis revealed no effect of age group on executive functions and game intelligence (see Table 1).

\section{Game time}

Moderate to large correlations with game time were present for game intelligence and cognitive flexibility whereby working memory capacity, cognition score and selective attention correlated small to moderately with game time. None of the other parameters showed meaningful correlations. After controlling for the age group, the correlations with game intelligence, cognitive flexibility, working memory capacity, cognition score and selective attention remained. In contrast the correlations with RIEA and acceleration (5 and 30 meters) became meaningful on a moderate to large level as well (see Table 2).

\section{Injuries}

\section{Contact Injuries}

Only 30-meter acceleration showed a small to moderate correlation with contact injuries $(n=13)$ whereas cognitive flexibility only showed a trend. None of the other parameters were meaningfully correlated. The correlation with 30-meter acceleration was reversed to a negative small to moderate correlation after controlling for the age group (see Table 3).

\section{Noncontact Injuries}

Small to moderate correlations with noncontact injuries $(n=22)$ were present for working memory capacity, inhibition, countermovement jump and squat jump. None of these correlations remained meaningful after controlling for age group whereas 30-meter acceleration showed a moderate to large and performance-IAT showed a negative small to moderate correlation (see Table 3).

Table 1

Partial correlations between executive functions and game intelligence whilst controlling for age group

\begin{tabular}{|llllll|}
\hline & Selective Attention & Working Memory & Cognitive Flexibility & Inhibition & Cognition Score \\
\hline $\begin{array}{l}\text { Game intelligence } \\
\text { Spearmans's } r\end{array}$ & 0.16 & 0.28 & 0.30 & 0.07 & 0.29 \\
$\mathrm{Cl}$ & $-0.02,0.33$ & $0.13,0.42$ & $0.15,0.44$ & $-0.09,0.22$ & $0.12,0.45$ \\
$n$ & 116 & 156 & 156 & 156 & 116 \\
\hline $\begin{array}{l}\text { Included } \\
\text { teams }\end{array}$ & U15-first team & U13-first team & U13-first team & U13-first team & U15-first team \\
\hline
\end{tabular}


Table 2

Partial correlations between executive functions, physiological abilities and game time whilst controlling for age group

\begin{tabular}{|c|c|c|c|c|c|c|c|c|c|c|c|c|c|}
\hline & $\begin{array}{l}\text { Game } \\
\text { Intelligence }\end{array}$ & $\begin{array}{l}\text { Selective } \\
\text { Attention }\end{array}$ & $\begin{array}{l}\text { Working } \\
\text { Memory }\end{array}$ & $\begin{array}{l}\text { Cognitive } \\
\text { Flexibility }\end{array}$ & Inhibition & $\begin{array}{l}\text { Cognition } \\
\text { Score }\end{array}$ & RIEA & $\begin{array}{l}\text { Sprint } \\
(5 \mathrm{M})\end{array}$ & $\begin{array}{l}\text { Sprint } \\
(30 \mathrm{~m})\end{array}$ & $\begin{array}{l}\text { Squat } \\
\text { Jump }\end{array}$ & $\begin{array}{l}\text { Counter } \\
\text { Movement } \\
\text { Jump }\end{array}$ & $\begin{array}{l}\text { Drop } \\
\text { Jump }\end{array}$ & $\begin{array}{l}\mathrm{Pe} \\
\mathrm{IA}^{-}\end{array}$ \\
\hline Game time & 0.42 & 0.22 & 0.29 & 0.34 & -0.17 & 0.29 & 0.32 & -0.34 & -0.37 & -0.01 & 0.04 & 0.14 & 0.1 \\
\hline $\begin{array}{l}\text { Spearmans's } \\
r\end{array}$ & $0.25,0.56$ & $\begin{array}{l}0.02 \\
0.40\end{array}$ & $\begin{array}{l}0.12 \\
0.44\end{array}$ & $\begin{array}{l}0.18 \\
0.48\end{array}$ & $\begin{array}{l}-0.33 \\
0.01\end{array}$ & $\begin{array}{l}0.10 \\
0.46\end{array}$ & $\begin{array}{l}0.10 \\
0.54\end{array}$ & $\begin{array}{l}-0.50 \\
-0.16\end{array}$ & $\begin{array}{l}-0.53 \\
-0.19\end{array}$ & $\begin{array}{l}-0.27 \\
0.25\end{array}$ & $-0.14,0.22$ & $\begin{array}{l}-0.04 \\
0.31\end{array}$ & -0 . \\
\hline $\begin{array}{l}\mathrm{Cl} \\
n\end{array}$ & 109 & 97 & 128 & 128 & 131 & 100 & 56 & 103 & 103 & 56 & 122 & 122 & 42 \\
\hline $\begin{array}{l}\text { Included } \\
\text { teams }\end{array}$ & $\begin{array}{l}\text { U13- } \\
\text { first team }\end{array}$ & $\begin{array}{l}\text { U15- } \\
\text { first } \\
\text { team }\end{array}$ & $\begin{array}{l}\text { U13- } \\
\text { first } \\
\text { team }\end{array}$ & $\begin{array}{l}\text { U13- } \\
\text { first team }\end{array}$ & $\begin{array}{l}\text { U13- } \\
\text { first } \\
\text { team }\end{array}$ & $\begin{array}{l}\text { U15- } \\
\text { first team }\end{array}$ & $\begin{array}{l}\text { U14- } \\
\text { U19 }\end{array}$ & $\begin{array}{l}\text { U13- } \\
\text { U23 }\end{array}$ & $\begin{array}{l}\text { U13- } \\
\text { U23 }\end{array}$ & $\begin{array}{l}\text { U15- } \\
\text { U19 }\end{array}$ & $\begin{array}{l}\text { U13- } \\
\text { first team }\end{array}$ & $\begin{array}{l}\text { U13- } \\
\text { first } \\
\text { team }\end{array}$ & $\begin{array}{l}\text { U1 } \\
\text { U1 }\end{array}$ \\
\hline
\end{tabular}

Note. RIEA: repeatedly intense exercise ability; IAT: individual anaerobic threshold

Table 3

Partial correlations between executive functions, physiological abilities and injuries whilst controlling for age group

\begin{tabular}{|c|c|c|c|c|c|c|c|c|c|c|c|c|c|}
\hline & $\begin{array}{l}\text { Game } \\
\text { Intelligence }\end{array}$ & $\begin{array}{l}\text { Selective } \\
\text { Attention }\end{array}$ & $\begin{array}{l}\text { Working } \\
\text { Memory }\end{array}$ & $\begin{array}{l}\text { Cognitive } \\
\text { Flexibility }\end{array}$ & Inhibition & $\begin{array}{l}\text { Cognition } \\
\text { Score }\end{array}$ & RIEA & $\begin{array}{l}\text { Sprint } \\
(5 M)\end{array}$ & $\begin{array}{l}\text { Sprint } \\
(30 \mathrm{~m})\end{array}$ & $\begin{array}{l}\text { Squat } \\
\text { Jump }\end{array}$ & $\begin{array}{l}\text { Counter } \\
\text { Movement } \\
\text { Jump }\end{array}$ & $\begin{array}{l}\text { Drop } \\
\text { Jump }\end{array}$ & $\begin{array}{l}P_{1} \\
\text { IA }\end{array}$ \\
\hline $\begin{array}{l}\text { Contact } \\
\text { Injury }\end{array}$ & -0.14 & 0.04 & -0.02 & 0.12 & -0.08 & -0.04 & 0.08 & -0.08 & -0.24 & 0.09 & 0.12 & 0.04 & -0 \\
\hline $\begin{array}{l}\text { Spearmans's } \\
r\end{array}$ & $-0.31,0.04$ & $\begin{array}{l}-0.15 \\
0.23\end{array}$ & $\begin{array}{l}-0.18 \\
0.15\end{array}$ & $\begin{array}{l}-0.05 \\
0.30\end{array}$ & $\begin{array}{l}-0.24 \\
0.09\end{array}$ & $\begin{array}{l}-0.23 \\
0.15\end{array}$ & $\begin{array}{l}-0.18 \\
0.33\end{array}$ & $\begin{array}{l}-0.26 \\
0.10\end{array}$ & $\begin{array}{l}-0.41 \\
-0.06\end{array}$ & $\begin{array}{l}-0.16 \\
0.33\end{array}$ & $-0.05,0.28$ & $\begin{array}{l}-0.13 \\
0.21\end{array}$ & -0 \\
\hline $\begin{array}{l}\mathrm{Cl} \\
n\end{array}$ & 122 & 108 & 141 & 141 & 141 & 108 & 61 & 115 & 115 & 66 & 134 & 134 & $4 \varepsilon$ \\
\hline $\begin{array}{l}\text { Noncontact } \\
\text { Injury }\end{array}$ & 0.08 & -0.14 & 0.11 & 0.05 & 0.16 & 0.14 & -0.20 & 0.11 & 0.35 & -0.12 & -0.19 & 0.16 & -0 \\
\hline $\begin{array}{l}\text { Spearmans's } \\
r\end{array}$ & $-0.10,0.25$ & $\begin{array}{l}-0.32 \\
0.10\end{array}$ & $\begin{array}{l}-0.10 \\
0.27\end{array}$ & $\begin{array}{l}-0.12 \\
0.21\end{array}$ & $\begin{array}{l}-0.01 \\
0.32\end{array}$ & $\begin{array}{l}-0.05 \\
0.32\end{array}$ & $\begin{array}{l}-0.43 \\
0.05\end{array}$ & $\begin{array}{l}-0.07 \\
0.30\end{array}$ & $\begin{array}{l}0.18 \\
0.50\end{array}$ & $\begin{array}{l}-0.35 \\
0.13\end{array}$ & $-0.35,0.02$ & $\begin{array}{l}-0.01 \\
0.32\end{array}$ & -0 \\
\hline $\begin{array}{l}\mathrm{Cl} \\
n\end{array}$ & 122 & 108 & 141 & 141 & 141 & 108 & 61 & 115 & 115 & 66 & 134 & 134 & $4 \varepsilon$ \\
\hline $\begin{array}{l}\text { Included } \\
\text { teams }\end{array}$ & $\begin{array}{l}\text { U13-first } \\
\text { team }\end{array}$ & $\begin{array}{l}\text { U15- } \\
\text { first } \\
\text { team }\end{array}$ & $\begin{array}{l}\text { U13- } \\
\text { first } \\
\text { team }\end{array}$ & $\begin{array}{l}\text { U13- } \\
\text { first team }\end{array}$ & $\begin{array}{l}\text { U13- } \\
\text { first } \\
\text { team }\end{array}$ & $\begin{array}{l}\text { U15- } \\
\text { first team }\end{array}$ & $\begin{array}{l}\text { U14- } \\
\text { U19 }\end{array}$ & $\begin{array}{l}\text { U13- } \\
\text { U23 }\end{array}$ & $\begin{array}{l}\text { U13- } \\
\text { U23 }\end{array}$ & $\begin{array}{l}\text { U15- } \\
\text { U19 }\end{array}$ & $\begin{array}{l}\text { U13- } \\
\text { first team }\end{array}$ & $\begin{array}{l}\text { U13- } \\
\text { first } \\
\text { team }\end{array}$ & $\begin{array}{l}U^{*} \\
U^{*}\end{array}$ \\
\hline
\end{tabular}

Note. RIEA: repeated intense exercise ability; IAT: individual anaerobic threshold; included teams: describes which teams contribute to the sample of each cor e.g. U13-first team indicates all teams starting from U13 up to the first team (U13,14,15,16,17,19,23, first team)

\section{General Discussion}

The prediction of talent is a crucial facet of domains in which humans need to thrive to high-performance in complex settings. Elite soccer is one of these settings where cognitive and physiological abilities are essential properties of the game whereas evidence on the predicational value of multidisciplinary performance data is scarce. The present results confirm our first hypothesis for the most part and propose that coach-rated game intelligence correlates small to moderately with the executive functions working memory and cognitive flexibility. In contrast, selective attention and inhibition had no relation. In agreement with the current investigation, a previous study ${ }^{16}$ found a moderate correlation of coach-rated game intelligence with design fluency, a test combining all three executive functions. But for the first time, the present study not only shows this relationship in adults but also in adolescents and children. Specifically, the current association seems to be present across all developmental stages (i.e. all tested age groups) as age had no meaningful effect on the correlation. This might significantly expand the findings of adult players and point at a fundamental linkage inherent to the soccer expertise at all included phases of age.

Further, it was proposed that specifically cognitive flexibility may contribute to game intelligence, which can be confirmed based on the present findings. Contrary to the current results, no correlation between game intelligence and working memory was found in the aforementioned study ${ }^{16}$. This may be based on the applied one-back working memory test as it is highly questionable if this very banal version which was only developed for concussion diagnostics is 
able to represent a similar level of complexity like soccer situations. Thus, especially cognitive flexibility and working memory capacity seem to be associated with game intelligence.

Regarding the second aim of the study, the present data indicate for the first time that better performance of executive functions (objective and coach-rated) is associated with an objective rating of successful soccer performance, i.e. game time, across all included ages (i.e. 12-34 years). More specifically, coach-rated game intelligence and cognitive flexibility represent moderate to large- and working memory capacity, cognition score and selective attention show small to moderate correlations with game time. As only inhibition showed no correlation the second hypothesis can be partially proved. These findings are in line with previous results on the interrelation of executive functions and superior performance in elite athletes ${ }^{13}$ and success in soccer as measured by scored goals, assists $^{14-16}$ and the acceptance into an elite soccer academy ${ }^{28}$.

But as an important extension, this evidence might also be enlarged as the measure of success in the present study may also be even more valid since game time is commonly used ${ }^{34}$ as it is achievable for players of all positions compared to goals and assists, which is more challenging to realize for defenders compared to strikers. Similarly to the correlation with game intelligence, it is remarkable that the unique relationship between executive functions and game time seems to exist across all teams as age had no meaningful effect on the correlation. Again, this could hint at a central association integral to soccer expertise at all developmental phases from age 12 up. Thus, these findings along with the relationship of game intelligence and executive functions across all age groups may be used as valuable insights into the current demands of high-performance in complex team sports like soccer as a kind of performanceneeds analysis ${ }^{41}$. Additionally, the findings enlarge the current understanding of the cognitive and physiological contribution to expertise and talent in certain developmental stages ${ }^{40,41,43,44}$. However, in contrast to prior studies suggesting the importance of inhibition for soccer players ${ }^{11,12}$ no association with game time was evident in the present study.

Furthermore, a key question is whether executive functions develop due to systematic exposure to high quantities and qualities of training (i.e. nurture) or whether this is a prerequisite to play on an elite level (i.e. nature). Recent findings of a large longitudinal study of elite soccer players question the nurture approach $^{33}$. Although the present results cannot completely resolve that debate, they may suggest that executive functions have a substantial relation to game intelligence and game time representing essential performance parameters of the soccer game. As an important extension to previous findings and contrary to Beavan et al. ${ }^{33}$, these associations are already present in the youngest age group indicating a low probability that this is solely based on natural predispositions. However, the difference in the applied executive function tests (e.g. no working memory or cognitive flexibility test in ${ }^{33}$ ) and age groups (i.e. no adult elite team $\mathrm{in}^{33}$ ) need to be considered which probably also contributes to the differing findings.

Furthermore, the results of the physiological performance data show that the covariate age had a considerable effect on the interactions of the physiological parameters RIEA and sprint with game time which could be explained by the fact that younger players' physiological capabilities are less developed compared to older players resulting in slower sprint times and lower endurance performance. By eliminating this age effect through partial correlation analysis, the underlying substantial association became noticeable. More specifically, the abilities RIEA and sprint ( 5 and 30 -meter time) were correlated on a moderate level with game time, confirming similar results of a previous review ${ }^{4}$ concerning their relation to success (i.e. the entrance to the next development stage of an elite youth academy). Thus, our third hypothesis can only be partially confirmed. This association seems intuitive as these skills are constantly mentioned as important performance indicators in soccer ${ }^{35,36}$. However, small differences between the current RIEA and endurance tests included in the review cannot be excluded and should therefore be considered.

None of the other physiological parameters showed meaningful associations except for drop jump and performance-IAT trends, which is contrary to prior review evidence ${ }^{4}$ and our hypothesis.

Consequently, the present results suggest that mainly the executive functions (except for inhibition) along with the physiological abilities sprint and RIEA contribute meaningfully to the game time of elite soccer players. As an essential addition, the current findings may partially answer the question of Beavan et al. $^{33}$ in terms of the predictive validity of domain-generic executive functions. However, the smaller sample sizes of the physiological parameters RIEA, squat jump and performance-IAT compared to the cognitive parameters also need to be considered.

This study also aimed to analyze the predictive capabilities of the multidisciplinary performance data in terms of future injuries. Concerning contact injuries, only the 30-meter sprint represents a meaningful negative correlation with contact injuries (i.e. small to moderate). The covariate age influenced this correlation (i.e. switching substantially from positive to negative), which may again be explained by the fact that the youngest teams (i.e. U13-U15) are slower than their older peers based on their developmental phase. As players of this age group sustained no injury at all, this incidence distorted the primary bivariate correlation before controlling for age. However, this counterintuitive result proposing that physically faster players sustain more contact injuries is not in agreement with previous literature suggesting that better sprint performance reduces the injury risk ${ }^{29}$ in adult elite soccer players and contradicts our fourth hypothesis. Yet, it is unclear whether the relationship of the study ${ }^{29}$ mentioned above is evident in all types of injuries (i.e. contact vs. non-contact). One could argue that faster players could have more duels and therefore contacts with other players resulting in an increased probability to sustain an injury. As $92 \%$ of the contact injuries are related to the lower limbs (see Appendix 6), this might indicate that the possible higher velocities of faster players may intensify the impact of those duel-contacts with opposing players and therefore increase the injury risk.

Additionally, the higher mechanical load in faster players could also contribute to this heightened risk ${ }^{37}$. Nevertheless, this is somewhat speculative as this association is the first of its kind to our knowledge. It also needs to be considered that no contact injuries occurred in the age groups U13-U15.

Moreover, although previous literature suggests that executive functions are related to injuries ${ }^{19}$ and injury-risks, ${ }^{17,18}$ no meaningful correlation with contactand non-contact injuries were observed in the present study, contrary to our fourth hypothesis. However, the different study designs of the previous 
investigations, including amateur athletes of various sports with predominantly controlled injury testing situations, may also explain this discrepancy. The current study analyzed the relationship between multidisciplinary performance data and elite athletes' injuries for the first time.

Regarding the second injury classification, "non-contact", a moderate to large correlation of sprint (30-meter) was present, indicating a lower non-contact injury incidence in faster players. This confirms previous studies indicating a lower injury risk in athletes with higher speed 29,32 and partially our hypothesis. $50 \%$ of all non-contact injuries (see Appendix 6) are related to musculature, which is in line with previous studies suggesting that well-developed sprinting-related muscles reduce the injury risk ${ }^{29,32}$. However, as a crucial expansion, present findings also show that the player's developmental phase influences this relationship.

Lastly, performance-IAT was negatively and small to moderately correlated with non-contact injuries suggesting that players with better anaerobic endurance performance are less likely to sustain a non-contact injury. Fatigue results in a higher injury risk based on lower coordination performance, among other factors due to decreased neuromuscular control ${ }^{38}$. Thus, as players with a better performance at the IAT get fatigued later, their injury risk may be reduced. Further, this association is also in line with the protective function of a well-developed cardiovascular and musculoskeletal system ${ }^{39}$. Again, the covariate age had a meaningful effect on both associations, similar to the contact injuries. However, the smaller sample size and the fact that the performance-IAT parameter was only present for the teams U16-U19 need to be considered.

Limitations of the current study should also be acknowledged. Although multidisciplinary performance data were used, it still does not capture the complex soccer game holistically as technical/tactical skills and psychological abilities (e.g. resilience) are missing. Additionally, while current research indicates that the included performance parameters are important properties of elite soccer, it is still possible that other parameters which were not analyzed may also contribute essentially to success in elite soccer. Lastly, due to the partially differing sample sizes contributing to the individual parameters (i.e. RIEA, IAT, SJ) these associations, of course, have a smaller precision compared to those with a larger sample size $>100$. This also leads to differing injury numbers as not all parameters are present for each team resulting in not considering injuries of that specific team where no data are available (e.g. for IAT).

In total, the age-independent and fundamental associations of executive functions with game intelligence and game time may extend previous findings and their importance for elite soccer expertise at all ages besides providing insights into current demands of high-performance in complex team sports like soccer as a performance-needs analysis ${ }^{41}$. The applied, unique multidisciplinary approach also highlights the crucial role of specific physiological abilities for success in soccer regarding game time and age-dependent injury avoidance. Future studies should also include technical/ tactical as well as psychological (i.e. resilience) skills to create a holistic approach to talent identification and the endeavor to track their future success. Further, more sophisticated parameters are needed to capture the multifaceted construct of success in a dynamic and complex setting like elite soccer.

\section{Methods}

\section{Participants}

A total of 172 male elite soccer players (12 - 34 years of age) from the youth academy's talent development program and the first team of a professional German soccer club were recruited. However, the dataset was not complete for all of the 172 players as some performance data were missing (i.e. CMJ, DJ: 4.6\%; game intelligence: $14.6 \%$; selective attention, cognition score: $23.7 \%$; acceleration (5 and 30-meter): $31.3 \%$; RIEA, SJ: 55\%; performance-IAT: $65.6 \%$ ) due to injuries, illness or other absence reasons. At the time of data collection, their teams played at the top level of their respective age group (U13, U14, U15, U16, $\mathrm{U17}$, U19, first team) or at the fourth-highest senior league (U23 team). Participants were not diagnosed with any behavioral, learning, or medical conditions that might influence cognitive abilities. Written informed consent was obtained from every participant or their legal guardian before commencing the tests. The study was carried out by the Helsinki Declaration of 1975 and was approved by the German Sport University Cologne's ethics committee.

\section{Procedure and Materials}

Data of cognitive tests were collected in a quiet room. The cognitive tests consisted of one 45-minute session and were conducted before a training session. A four task-battery was used to explore the performance of executive functions. Each task started with practice trials in the beginning and is described below. Fixed test order was used, a standard method in neuropsychological assessment: 1) working memory, 2) cognitive flexibility, 3) inhibition and 4) multipleobject tracking. All participants were asked to sit in a comfortable position leaning against the backrest of the chair so that the screen distance was the same for all the players. Physiological performance data were collected on a separate session and are described in detail below. All cognitive and physical data were obtained during the first three weeks of the season 2020/201. The reason for the differing sample sizes contributing to the individual correlations is that not every test was conducted in every age group. Leaders of the test batteries were experienced sport scientists and athletic coaches.

\section{Executive Function Tests}

Working Memory Capacity (WMC) was measured using the well-established working memory span test by Conway, Kane, Bunting, Hambrick, Wilhelm \& Engle (2005). It measures the player's skill to direct attention toward the present task without getting distracted by other thoughts. More precisely, a counting span task was applied (see Kane et al., 2004 for a detailed description), as this processing task's simplicity allows a widespread application (Conway et al., 2005). The instructions were presented as written text on the computer screen. The counting span task was to count specific shapes among distractors and afterward remembering the count totals for later recall. Every stimulus image included randomly arranged dark blue circles, green circles, and dark blue squares. The task of the participants was counting the dark blue circles and then the total count at the end. A recall mask was presented after $2-7$ stimulus images into which participants had to fill their memorized count totals in the exact order they had been illustrated in. The participants counting span score 
was a partial credit load score (cf. Conway et al., 2005), which represents the sum of all correctly recalled elements - whereby a correctly remembered piece from a set containing two elements receives 2 points, and a correctly remembered element from a set with 7 items receives 7 points - divided by the maximum possible score. The test included 15 trials. The dependent measure was the score of correctly memorized objects in percentage representing the working memory capacity (Scharfen \& Memmert, 2019b).

Cognitive Flexibility (CF) was measured with the Trail Making Test (TMT) including two parts (A and B) (Sánchez-Cubillo, Periáñez, Adrover-Roig, RodríguezSánchez Ríos-Lago, Tirapu \& Barceló, 2009). The TMT-A is commonly used to measure visuo-perceptual abilities whereas TMT-B is used to assess cognitive flexibility (Crowe, 1998). A smaller B-A difference indicates better cognitive flexibility (for a detailed description see Huijgen et al., 2015). A validated tablet version of the TMT was used, which is congruent with the traditional pen-paper version (Delbaere \& Lord, 2015).

Inhibition was assessed with a computer-based language-independent Stop-Signal Task (SST) from the Cambridge Neuropsychological Test of Automated Battery (CANTAB; Cambridge Cognition 2019). The participant's response inhibition was essentially measured by asking them to take part in two opposing tasks: a Go task and a Stop task. They were instructed to press a left-hand button when an arrow occurred which pointed to the left, and a right-hand button when the arrow pointed right (i.e. Go task in $75 \%$ of trials). Additionally, they were instructed to inhibit the response and not press any buttons when they heard an auditory 'beep' signal (i.e. Stop task in $25 \%$ of trials). The onset of the 'beep' signal varied depending on a staircase protocol (i.e. either decreased or increased) based on their performance in the previous trial. The dependent variable is the Stop-Signal Reaction time (SSRT) whereby a higher value indicates poorer inhibitory control (Fogel et al., 2019). More detailed information about the test protocol has been described previously (Fogel et al., 2019).

Multiple-Object Tracking was analyzed with the NeuroTracker 3D-MOT task with the NeuroTracker ${ }^{\text {TM }}$ Core Program by CogniSens Athletics Inc. from the University of Montreal. The program was displayed on a wall via a video projector. The task included eight balls of which four are marked for two seconds. Then, the four marked balls needed to be tracked for 8 seconds. Afterward, the tracked balls are required to be stated in to analyze the performance. Other settings were the same as in Faubert (2013).

The value Cognition-Total was calculated by adding the z-standardized scores of all cognitive tests and dividing the sum by the number of included tests.

\section{Assessment of game intelligence}

The head coaches of each team (i.e. eight in total) were asked to judge their players' game intelligence compared to what they perceive as the average level of their respective league and age group (e.g. German Bundesliga for the first team). Similar to a previous study, a stanine-scale was used with 5 representing the average of their league and age group, 1 is the lowest and 9 the highest value ${ }^{16}$. To ensure the objectivity of this game intelligence evaluation the ratings of the heads of the respective age groups were analyzed as well. A high degree of inter-rater reliability was present as the average measure ICC was .82.

\section{Physiological Tests}

The Individual Anaerobic Threshold (IAT) represents the maximal exercise intensity that can be sustained for a continued period without lactate substantially building up in the athlete's blood. IAT was assessed with a staircase-field test following the protocol of Faude et al. (2014) with the difference that the protocol was the same for every player - initial speed was $9 \mathrm{~km} / \mathrm{h}$ and speed of the last stage was $16.5 \mathrm{~km} / \mathrm{h}$.

Vertical jump performance was assessed by (1) countermovement jump (CMJ), (2) squat jump (SJ), (3) drop jump (DJ) (box height = $35 \mathrm{~cm}$ ) on a contact mat (Smart Jump; Smart speed, Fusion Sport, Australia). The protocol of Faude et al. (2014) was applied except for jumping with arm usage to represent soccerspecific demands. Dependent measures for CMJ and SJ were height and a quotient for DJ (i.e. DJQ), the calculated relation of height and contact time.

Sprint performance was assessed with infrared timing gates (Smart speed, Fusion Sport, Australia). The protocol of Faude et al. (2014) was applied with a starting line 0.5 meters behind the first timing gate whereby resting time was 3 minutes and only the best 5- and 30-meter time was included as the dependent variable.

The repeated intense exercise ability (RIEA) was assessed with the YoYo intermittent recovery test level 1 by following the protocol of Krustrup et al. (2003). All tests were applied on a soccer pitch with artificial grass, and players conducted a standardized warm-up before starting the test. Excellent reliability and validity are stated for this test (Krustrup et al., 2003; Deprez et al., 2014). The dependent variable is the maximal reached performance stage.

\section{Game time}

Game time was evaluated during the beginning of the season 2020/21 till the end of October, resulting in a minimum of three and a maximum of eight games per team. The reason for this cut-off was the ceasing of the regular soccer leagues. Game time for each player was noted by using the club-internal data records. To gain a standardized game time score for each player independent of age group and their overall differing game durations, the percentage of the maximal possible game time was calculated. This maximal possible game-time percentage was reduced to $0 \%$ when the absence reason of a player was an injury or illness, whereas the score remained at $100 \%$ if the lack was due to performance reasons.

\section{Injury incidences}


Injuries were diagnosed by the club's medical staff and recorded if that injury prevented a player from taking a full part in all training and match-play activities typically planned for that day and prevented participation for a period greater than $24 \mathrm{~h}$. This reflects the definition of Brooks et al. ${ }^{30}$ and conforms to the consensus definition for team sport athletes ${ }^{31}$. The diagnosed injuries were further classified in terms of mechanism (i.e., contact or non-contact), injury type and location which is also based on the consensus definition for team sport athletes ${ }^{31}$.

\section{Statistical Analysis}

Data were analyzed using IBM SPSS Statistics 26.0.0. Current recommendations to focus on estimation for best reporting and analysis practice were followed instead of conducting null-hypothesis significance tests (Cumming, 2014); effect-sizes with 95\% confidence intervals are reported. Not all variables were normally distributed, as assessed by Shapiro-Wilk's test $(p<0.05)$. Therefore, the Spearman's correlation coefficient test was used to investigate the correlation between the player's cognitive, physiological performance and game intelligence, game time and injuries. Correlation coefficients (Spearman's r) of $0.1,0.3$, and 0.5 represent small, moderate, and large effect size estimates, respectively (Cohen, 1998). To confirm that other factors did not confound the findings, we also performed a non-parametric partial correlation analysis ${ }^{27}$, which controlled the age group.

\section{Declarations}

\section{Declaration of interest}

The authors declare no conflicting interests.

\section{Data availability}

The datasets generated and analyzed during the current study are available from the corresponding author on reasonable request.

\section{Funding}

No special funding was received.

\section{References}

1. Coulson-Thomas, C. (2012). Talent management and building high performance organisations. Industrial and Commercial Training, 44(7), 429-436. https://doi.org/10.1108/00197851211268027

2. (FIFA Communications Division, Information Services., 2007)

3. Huijgen, B. C. H., Elferink-Gemser, M. T., Ali, A., \& Visscher, C. (2013). Soccer Skill Development in Talented Players. International Journal of Sports Medicine, 34, 720-726.

4. Murr, D., Raabe, J., \& Höner, O. (2018). The prognostic value of physiological and physical characteristics in youth soccer: A systematic review. European Journal of Sport Science, 18(1), 62-74. https://doi.org/10.1080/17461391.2017.1386719

5. Williams, A. M., Ford, P. R., \& Drust, B. (2020). Talent identification and development in soccer since the millennium. Journal of Sports Sciences, 38(1112), 1199-1210. https://doi.org/10.1080/02640414.2020.1766647

6. Murr, D., Feichtinger, P., Larkin, P., O’Connor, D., \& Höner, O. (2018). Psychological talent predictors in youth soccer: A systematic review of the prognostic relevance of psychomotor, perceptual-cognitive and personality-related factors. PLoS ONE, 13(10). https://doi.org/10.1371/journal.pone.0205337

7. Ivarsson, A., Kilhage-Persson, A., Martindale, R., Priestley, D., Huijgen, B., Ardern, C., \& McCall, A. (2020). Psychological factors and future performance of football players: A systematic review with meta-analysis. Journal of Science and Medicine in Sport, 23(4), 415-420.

https://doi.org/10.1016/j.jsams.2019.10.021

8. Friedman, N. P., Miyake, A., Corley, R. P., Young, S. E., DeFries, J. C., \& Hewitt, J. K. (2006). Not All Executive Functions Are Related to Intelligence. Psychological Science, 17(2), 172-179. https://doi.org/10.1111/j.1467-9280.2006.01681.x

9. Miyake, A., \& Friedman, N. P. (2012). The nature and organization of individual differences in executive functions: Four general conclusions. Current Directions in Psychological Science, 21(1), 8-14. https://doi.org/10.1177/0963721411429458

10. Huijgen, B. C. H., Leemhuis, S., Kok, N. M., Verburgh, L., Oosterlaan, J., Elferink-Gemser, M. T., \& Visscher, C. (2015). Cognitive functions in elite and sub-elite youth soccer players aged 13 to 17 years. PLOS ONE, 1O(12), 1-13. https://doi.org/10.1371/journal.pone.0144580

11. Verburgh, L., Scherder, E. J. A., Van Lange, P. A., \& Oosterlaan, J. (2014). Executive Functioning in Highly Talented Soccer Players. PLoS ONE, $9(3), 91254$. https://doi.org/10.1371/journal.pone.0091254

12. Verburgh, L., Scherder, E. J. A., Van Lange, P. A. M., \& Oosterlaan, J. (2016). Do elite and amateur soccer players outperform non-athletes on neurocognitive functioning? A study among 8-12 year old children. PLOS ONE, 11(12). https://doi.org/10.1371/journal.pone.0165741

13. Scharfen, H. E., \& Memmert, D. (2019). Measurement of cognitive functions in experts and elite athletes: A meta-analytic review. Applied Cognitive Psychology. https://doi.org/10.1002/acp.3526 
14. Vestberg, T., Gustafson, R., Maurex, L., Ingvar, M., \& Petrovic, P. (2012). Executive Functions Predict the Success of Top-Soccer Players. PLoS ONE, 7(4), 34731. https://doi.org/10.1371/journal.pone.0034731

15. Vestberg, T., Reinebo, G., Maurex, L., Ingvar, M., \& Petrovic, P. (2017). Core executive functions are associated with success in young elite soccer players. PloS One, 12(2), e0170845. https://doi.org/10.1371/journal.pone.0170845

16. Vestberg, T., Jafari, R., Almeida, R., Maurex, L., Ingvar, M., \& Petrovic, P. (2020). Level of play and coach-rated game intelligence are related to performance on design fluency in elite soccer players. Scientific Reports, 10(1), 1-10. https://doi.org/10.1038/s41598-02066180-w

17. Giesche, F., Wilke, J., Engeroff, T., Niederer, D., Hohmann, H., Vogt, L., \& Banzer, W. (2020). Are biomechanical stability deficits during unplanned single-leg landings related to specific markers of cognitive function? Journal of Science and Medicine in Sport, 23(1), 82-88.

https://doi.org/10.1016/j.jsams.2019.09.003

18. Monfort, S. M., Pradarelli, J. J., Grooms, D. R., Hutchison, K. A., Onate, J. A., \& Chaudhari, A. M. W. (2019). Visual-Spatial Memory Deficits Are Related to Increased Knee Valgus Angle During a Sport-Specific Sidestep Cut. American Journal of Sports Medicine, 47(6), 1488-1495. https://doi.org/10.1177/0363546519834544

19. Swanik, C. B., Covassin, T., Stearne, D. J., \& Schatz, P. (2007). The relationship between neurocognitive function and noncontact anterior cruciate ligament injuries. American Journal of Sports Medicine, 35(6), 943-948. https://doi.org/10.1177/0363546507299532

20. Ivarsson, A., Johnson, U., Andersen, M. B., Tranaeus, U., Stenling, A., \& Lindwall, M. (2017). Psychosocial Factors and Sport Injuries: Meta-analyses for Prediction and Prevention. Sports Medicine, 47(2), 353-365. https://doi.org/10.1007/s40279-016-0578-x

21. Faubert, J. (2013). Professional athletes have extraordinary skills for rapidly learning complex and neutral dynamic visual scenes. Scientific Reports, 3. https://doi.org/10.1038/srep01154

22. Romeas, T., Guldner, A., \& Faubert, J. (2016). 3D-Multiple Object Tracking training task improves passing decision-making accuracy in soccer players. Psychology of Sport and Exercise, 22, 1-9. https://doi.org/10.1016/j.psychsport.2015.06.002

23. Unnithan, V., White, J., Georgiou, A., Iga, J., \& Drust, B. (2012). Talent identification in youth soccer. Journal of Sports Sciences, 30(15), $1719-1726$. https://doi.org/10.1080/02640414.2012.731515

24. Waldron, M., \& Murphy, A. (2013). A comparison of physical abilities and match performance characteristics among elite and subelite under-14 soccer players. Pediatric Exercise Science, 25(3), 423-434. http://www.ncbi.nlm.nih.gov/pubmed/23877584

25. Abade, E. A., Gonçalves, B. V., Silva, A. M., Leite, N. M., Castagna, C., \& Sampaio, J. E. (2014). Classifying Young Soccer Players by Training Performances. Perceptual and Motor Skills, 119(3), 971-984. https://doi.org/10.2466/10.25.PMS.119c31z8

26. Bangsbo, J., laia, F. M., \& Krustrup, P. (2008). The Yo-Yo Intermittent Recovery Test A Useful Tool for Evaluation of Physical Performance in Intermittent Sports Jens. Sports Medicine, 38(1), 37-51.

27. Conover, W.J. (1999). Practical Nonparametric Statistics (3rd Ed.). New York: Wiley, (p. 327-328).

28. Sakamoto, S., Takeuchi, H., Ihara, N., Ligao, B., \& Suzukawa, K. (2018). Possible requirement of executive functions for high performance in soccer. PLoS ONE, 13(8). https://doi.org/10.1371/journal.pone.0201871

29. Malone, S., Owen, A., Mendes, B., Hughes, B., Collins, K., \& Gabbett, T. J. (2018). High-speed running and sprinting as an injury risk factor in soccer: Can well-developed physical qualities reduce the risk? Journal of Science and Medicine in Sport, 21(3), 257-262. https://doi.org/10.1016/j.jsams.2017.05.016

30. Brooks, J. H. M., Fuller, C. W., Kemp, S. P. T., \& Reddin, D. B. (2005). Epidemiology of injuries in English professional rugby union: Part 1 match injuries. British Journal of Sports Medicine, 39(10), 757-766. https://doi.org/10.1136/bjsm.2005.018135

31. Fuller, C. W., Molloy, M. G., Bagate, C., Bahr, R., Brooks, J. H. M., Donson, H., Kemp, S. P. T., McCrory, P., Mclntosh, A. S., Meeuwisse, W. H., Quarrie, K. L., Raftery, M., \& Wiley, P. (2007). Consensus statement on injury definitions and data collection procedures for studies of injuries in Football. Clinical Journal of Sport Medicine, 17(3), 177-181. https://doi.org/10.1097/JSM.0b013e31803220b3

32. Malone, S., Hughes, B., Doran, D. A., Collins, K., \& Gabbett, T. J. (2019). Can the workload-injury relationship be moderated by improved strength, speed and repeated-sprint qualities? Journal of Science and Medicine in Sport, 22(1), 29-34. https://doi.org/10.1016/j.jsams.2018.01.010

33. Beavan, A., Chin, V., Ryan, L. M., Spielmann, J., Mayer, J., Skorski, S., Meyer, T., \& Fransen, J. (2020). A longitudinal analysis of the executive functions in high-level soccer players. Journal of Sport and Exercise Psychology, 42(5), 349-357. https://doi.org/10.1123/JSEP.2019-0312

34. Rumbold, J., Fletcher, D., \& Daniels, K. (2020). An experience sampling study of organizational stress processes and future playing time in professional sport. Journal of Sports Sciences, 38(5), 559-567. https://doi.org/10.1080/02640414.2020.1717302

35. Reilly, T., Williams, A. M., Nevill, A., \& Franks, A. (2000). A multidisciplinary approach to talent identification in soccer. Journal of Sports Sciences, 18(9), 695-702. https://doi.org/10.1080/02640410050120078

36. Oone, J. A. N. B., Aeyens, R. O. E. L. V, Teyaert, A. D. S., Ossche, L. U. C. V. A. B., \& Ourgois, J. A. N. B. (2012). Physical fitness of elite belgian soccer players by player position. Journal of Strength and Conditioning Research, 26(8), 2051-2057

37. Beato, M., \& Drust, B. (2020). Acceleration intensity is an important contributor to the external and internal training load demands of repeated sprint exercises in soccer players. Research in Sports Medicine, 29(1), 67-76. https://doi.org/10.1080/15438627.2020.1743993

38. Huygaerts, S., Cos, F., Cohen, D. D., Calleja-González, J., Guitart, M., Blazevich, A. J., \& Alcaraz, P. E. (2020). Mechanisms of Hamstring Strain Injury: Interactions between Fatigue, Muscle Activation and Function. Sports, 8(5), 65. https://doi.org/10.3390/sports8050065

39. Gabbett, T. J. (2016). The training-injury prevention paradox: Should athletes be training smarter and harder? British Journal of Sports Medicine, 50(5), 273-280. https://doi.org/10.1136/bjsports-2015-095788

40. Johnston, K., Wattie, N., Schorer, J., \& Baker, J. (2018). Talent Identification in Sport: A Systematic Review. Sports Medicine, 48(1), 97-109. https://doi.org/10.1007/s40279-017-0803-2 
41. Baker, J., Wilson, S., Johnston, K., Dehghansai, N., Koenigsberg, A., de Vegt, S., \& Wattie, N. (2020). Talent Research in Sport 1990-2018: A Scoping Review. Frontiers in Psychology, 11(November). https://doi.org/10.3389/fpsyg.2020.607710

42. Rees, T., Hardy, L., Güllich, A., Abernethy, B., Côté, J., Woodman, T., Montgomery, H., Laing, S., \& Warr, C. (2016). The Great British Medalists Project: A Review of Current Knowledge on the Development of the World's Best Sporting Talent. Sports Medicine, 46(8), 1041-1058. https://doi.org/10.1007/s40279-016-0476-2

43. Till, K., \& Baker, J. (2020). Challenges and [Possible] Solutions to Optimizing Talent Identification and Development in Sport. Frontiers in Psychology, 11(April), 1-14. https://doi.org/10.3389/fpsyg.2020.00664

44. Baker, J., Wattie, N., \& Schorer, J. (2019). A proposed conceptualization of talent in sport: The first step in a long and winding road. Psychology of Sport and Exercise, 43, 27-33. https://doi.org/10.1016/j.psychsport.2018.12.016

45. Vaeyens, R., Güllich, A., Warr, C. R., \& Philippaerts, R. (2009). Talent identification and promotion programmes of olympic athletes. Journal of Sports Sciences, 27(13), 1367-1380. https://doi.org/10.1080/0264041090311097

\section{Supplementary Files}

This is a list of supplementary files associated with this preprint. Click to download.

- supplementaryFiles.docx

- AppendixSR.docx 